\title{
Comunicação
}

[Communication]

\section{Resistência antimicrobiana em Salmonella Enteritidis isoladas de amostras clínicas e ambientais de frangos de corte e matrizes pesadas}

\author{
[Antimicrobial resistance in Salmonella Enteritidis isolated from clinical and environmental \\ broiler chickens and breeders broiler] \\ A.R. Ribeiro ${ }^{1 *}$, A. Kellermann ${ }^{1}$, L.R. Santos $^{2}$, V.P. Nascimento ${ }^{1}$ \\ ${ }^{1}$ Faculdade de Veterinária - (CDPA) - UFRGS \\ Rua Bento Gonçalves, 8824 \\ 91540-000 - Porto Alegre, RS \\ ${ }^{2}$ Faculdade de Agronomia e Medicina Veterinária - UPF - Passo Fundo, RS
}

No passado, Salmonella Pullorum e Salmonella Gallinarum foram as principais causadoras de problemas nas galinhas; porém, devido a programas de erradicação, elas foram praticamente eliminadas (Pomeroy e Nagaraja, 1991; Snoeynbos e Willians, 1991). Baumler et al. (2000) postularam que, devido à erradicação dessas salmonelas, abriu-se um nicho que foi ocupado por Salmonella Enteritidis, uma vez que tanto esta como Salmonella Gallinarum e Salmonella Pullorum pertencem ao sorogrupo D1, indicando similaridade de cadeia lipopolissacarídica.

No Brasil, dentre os microrganismos isolados de aves portadoras e doentes provenientes de diversas regiões durante o período de 1961 a 1991, predominaram Salmonella Gallinarum, Salmonella Pullorum, Salmonella Typhimurium, Salmonella Heidelberg, Salmonella Enteritidis e Salmonella Infantis (Hofer et al., 1997). Contudo, em amostras isoladas no qüinqüênio 1992 - 1996, predominou Salmonella Enteritidis, (Solari et al., 1997), fato este que se manteve entre julho de 1997 e dezembro de 2004 (Kanashiro et al., 2005).

Em medicina veterinária, os agentes antimicrobianos são utilizados de forma terapêutica, metafilática, profilática e como promotores de crescimento (Scharwz et al., 2001). Como exemplo de forma preventiva, tem- se a apregoada por Goren (1994), que recomenda combinar medicamentos e microbiota intestinal, como ferramenta para controle de Salmonella Enteritidis em aves. Situações como estas têm provocado, nesses microrganismos, aumento da resistência a agentes antimicrobianos.

O presente estudo foi realizado com a finalidade de avaliar a resistência a agentes antimicrobianos de 79 amostras de $S$. Enteritidis isoladas de espécimes clínicas de frangos de corte e matrizes pesadas $(n=39)$ e de amostras do ambiente (swabs de arrasto), provenientes de aviários de frango de corte e de matrizes pesadas $(n=40)$, produzidos na região Sul do Brasil, nos anos de $1999(n=31), 2000 \quad(n=29)$ e $2001 \quad(n=19)$, utilizando-se metodologia recomendada pelo Ministério da Agricultura, Pecuária e Abastecimento para detecção de Salmonella spp. (Brasil, 1995). A caracterização antigênica e a identificação do sorovar foram realizadas pelo Laboratório de Bactérias Entéricas do Instituto Oswaldo Cruz, Rio de Janeiro.

As amostras de $S$. Enteritidis foram submetidas a testes de sensibilidade conforme técnica do NCCLS (Performance..., 2003), com a utilização de discos impregnados com os seguintes agentes antimicrobianos: ampicilina, $10 \mu \mathrm{g}$; ácido nalidíxico, $\quad 30 \mu \mathrm{g} ; \quad$ ciprofloxacina, $5 \mu$; cloranfenicol, $30 \mu$; enrofloxacina, $5 \mu$; gentamicina, $10 \mu ; \quad$ canamicina, $30 \mu$;

Recebido em 8 de outubro de 2007

Aceito em 25 de agosto de 2008

E-mail: aldemir_r@yahoo.com 
nitrofurantoína, $300 \mu$; norfloxacina, $10 \mu$; polimixina B, 300IU; estreptomicina, $10 \mu \mathrm{g}$ e

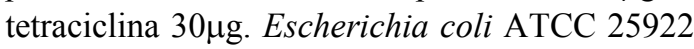
foi utilizada como amostra de referência.

Os resultados indicam que $82,3 \%(65 / 79)$ das amostras de $S$. Enteritidis foram resistentes a um ou mais agentes antimicrobianos utilizados, apresentando 19 padrões de resistência (Tab. 1). Dentre as 65 amostras de $S$. Enteritidis resistentes, $43(66,1 \%)$ apresentaram resistência a dois ou mais agentes antimicrobianos e 22 $(33,8 \%)$ a somente um. Destas, 16 foram resistentes à tetraciclina, três ao ácido nalidíxico, duas à nitrofurantoína e uma à gentamicina.

Resistência à tetraciclina foi observada em 53 amostras (67,1\%) de $S$. Enteritidis (Tab. 2). Esse resultado é mais alto que o encontrado por Antunes et al. (2003), em amostras de Salmonella spp. isoladas de produtos de aves no Porto, Portugal (36\%), porém menor que os obtidos por Cortez et al. (2006) em amostras de Salmonella spp. isoladas de abatedouros de aves no estado de São Paulo (72,4\%), por Ribeiro et al. (2007), em Salmonella spp. isoladas de cortes de frango no estado do Rio Grande do Sul (84\%).
Tabela 1. Distribuição dos padrões de resistência de 79 amostras de Salmonella Enteritidis isoladas de aves e ambientes de aviários na Região Sul do Brasil

\begin{tabular}{lc} 
Padrão de resistência* & $\mathrm{N}^{\mathrm{O}}$ \\
\hline Gen & 1 \\
Nal & 3 \\
Nit & 2 \\
Tet & 16 \\
Gen, Str & 2 \\
Gen, Tet & 1 \\
Nal, Tet & 5 \\
Nit, Tet & 13 \\
Enr, Nal, Tet & 3 \\
Gen, Nal, Str & 2 \\
Gen, Str, Tet & 4 \\
Nal, Nit, Tet & 3 \\
Amp, Gen, Nit, Str & 1 \\
Cip, Enr, Nal, Tet & 3 \\
Gen, Nal, Nit, Str & 1 \\
Gen, Nal, Str, Tet & 3 \\
Gen, Nit, Str, Tet & 1 \\
Enr, Gen, Kan, Nal, Nal, Str, Tet & 1 \\
\hline
\end{tabular}

*14 amostras foram sensíveis aos antimicrobianos testados.

Gen: gentamicina; Nal: ácido nalidíxico; Nit: nitrofurantóina; Tet: tetraciclina; Str: estroptomicina; Enr: enrofloxacina; Amp: ampicilina; Cip: ciprofloxacina; Kan: kanamicina

Tabela 2. Resistência antimicrobiana em amostras de $S$. Enteritidis isoladas de aves e ambientes de aviários da Região Sul do Brasil

\begin{tabular}{|c|c|c|c|c|c|c|c|c|c|c|c|c|c|}
\hline \multirow{2}{*}{ Ano } & \multirow{2}{*}{$\begin{array}{c}\text { Amostras } \\
\text { testadas }\end{array}$} & \multicolumn{12}{|c|}{ Amostras resistentes (\%) } \\
\hline & & Amp & Cip & Chl & Enr & Gen & Kan & $\mathrm{Nal}$ & Nit & Nor & PB & Str & Tet \\
\hline 1999 & 32 & $\begin{array}{c}1 \\
(3,1)\end{array}$ & 0 & 0 & 0 & $\begin{array}{c}5 \\
(15,6)\end{array}$ & 0 & $\begin{array}{c}6 \\
(18,7)\end{array}$ & $\begin{array}{c}10 \\
(31,2)\end{array}$ & 0 & 0 & $\begin{array}{c}5 \\
(15,6)\end{array}$ & $\begin{array}{c}24 \\
(75)\end{array}$ \\
\hline 2000 & 28 & 0 & $\begin{array}{c}1 \\
(3,6)\end{array}$ & 0 & $\begin{array}{c}3 \\
(10,7)\end{array}$ & $\begin{array}{c}5 \\
(17,8)\end{array}$ & 0 & $\begin{array}{c}8 \\
(28,5)\end{array}$ & $\begin{array}{c}5 \\
(17,8)\end{array}$ & 0 & 0 & $\begin{array}{c}5 \\
(17,8)\end{array}$ & $\begin{array}{c}17 \\
(60,7)\end{array}$ \\
\hline 2001 & 19 & 0 & $\begin{array}{c}2 \\
(10,5)\end{array}$ & 0 & $\begin{array}{c}4 \\
(21)\end{array}$ & $\begin{array}{c}8 \\
(42,1)\end{array}$ & $\begin{array}{c}1 \\
(5,2)\end{array}$ & $\begin{array}{c}10 \\
(52,6)\end{array}$ & $\begin{array}{c}6 \\
(31,6)\end{array}$ & 0 & 0 & $\begin{array}{c}6 \\
(31,6)\end{array}$ & $\begin{array}{c}12 \\
(63,1)\end{array}$ \\
\hline Total & 79 & $\begin{array}{c}1 \\
(1,2)\end{array}$ & $\begin{array}{c}3 \\
(3,8)\end{array}$ & 0 & $\begin{array}{c}7 \\
(8.8)\end{array}$ & $\begin{array}{c}18 \\
(22,8)\end{array}$ & $\begin{array}{c}1 \\
(1,2)\end{array}$ & $\begin{array}{c}24 \\
(30,4)\end{array}$ & $\begin{array}{c}21 \\
(26,6)\end{array}$ & 0 & 0 & $\begin{array}{c}16 \\
(20,2)\end{array}$ & $\begin{array}{c}53 \\
(67,1)\end{array}$ \\
\hline
\end{tabular}

Amp: ampicilina; Cip: ciprofloxacina; Chl: cloranfenicol; Enr: enrofloxacina; Gen: gentamicina; Kan: kanamicina;

Nal: ácido nalidíxico; Nit: nitrofurantoína; Nor: norfloxacina; PB: polimixina B; Str: estreptomicina; Tet: tetraciclina

Com relação à estreptomicina, $20,2 \%$ das amostras foram resistentes, resultado menor que os $25,4 \%$ relatados por Bokanyi Jr. et al. (1990) em Salmonella spp. isoladas de carcaças e cortes de aves nos Estados Unidos.

A elevada ocorrência de amostras resistentes à tetraciclina e à estreptomicina pode ser explicada pela difusão dos genes de resistência $\operatorname{str} A-s t r B$ e tet(A), como observado por Pezzella et al.
(2004), em estudo com amostras de Salmonella spp. isoladas de animais na Itália.

Encontrou-se baixa resistência à nitrofurantoína, $26,6 \%$, quando comparada com os $95 \%$ descritos por Cardoso et al. (2006) em amostras de $S$. Enteritidis isoladas de carcaças de frango entre maio de 1995 e abril de 1996, no Rio Grande do Sul. 
Resistência aos aminoglicosídeos, gentamicina e canamicina, apresentada pelas amostras de $S$. Enteritidis, foram $21,5 \%$ e $1,2 \%$, respectivamente. No Canadá, Poppe et al. (1996), em Salmonella spp. isoladas de aves, verificaram que somente $7,7 \%$ delas apresentaram resistência à gentamicina e, com relação à canamicina, Carramiñana et al. (2004) observaram somente $2,8 \%$ de resistência de amostras $S$. Enteritidis isoladas em um matadouro frigorífico na Espanha.

A resistência às quinolonas é determinada, fundamentalmente, por mecanismos mediados por alterações no cromossomo, alteração nos sítios de ligação da DNA gyrase e diminuição no acúmulo do agente no interior da bactéria (Piddock, 2002; Ruiz, 2003).

Elementos móveis que carreiam o gene $q n r$ também têm sido descritos como responsáveis por conferir resistência às quinolonas, sendo que estes apresentam um agravante, o fato de terem o potencial de transferir de forma horizontal os genes de resistência (Ruiz, 2003).
Portanto, os resultados obtidos para o ácido nalidíxico $(30,4 \%)$, enrofloxacina $(8,8 \%)$ e ciprofloxacina $(2,5 \%)$ são preocupantes, pois estudos recentes têm detectado aumento de amostras de Salmonella spp. resistentes às quinolonas na Alemanha (Malorny et al., 1999), Inglaterra e País de Gales (Threlfall et al., 2000) e Espanha (Marinón et al., 2004). Não foram observadas amostras resistentes à norfloxacina.

Pode-se concluir que há alta porcentagem de amostras de $S$. Enteritidis resistentes à tetraciclina, que o monitoramento contínuo é necessário na indústria avícola e que há necessidade de uso responsável dos agentes antimicrobianos, baseado na compreensão da ecologia da resistência, da transmissão de bactéria resistente e de genes de resistência e da relação entre o uso do antimicrobiano e o aumento da resistência (Turnidge, 2004).

Palavras-chave: ave, Salmonella Enteritidis, resistência antimicrobiana

\begin{abstract}
The antimicrobial resistance of Salmonella Enteritidis strains isolated from clinical and environmental poultry samples in the Southern Brazil during the years of 1999, 2000 and 2001 was evaluated. Among the 79 isolated samples, 64 (81\%) were resistant to at least one of the antimicrobial agents tested, showing 22 different resistance patterns. Tetracycline showed the highest percentage (64,5\%) of resistance among the antimicrobial agents used. Resistance to drugs at different levels was found as the following: ampicillin (1.2\%), kanamycin (1.2\%), ciprofloxacin (2.5\%), enrofloxacin (8.8\%), gentamicin (21.5\%), streptomycin (20.2\%), nitrofurantoin (26.6\%), and nalidixic acid (30.4\%). None of the S. Enteritidis strains were resistant to chloramphenicol, norfloxacin, and polimycin B. Among the $64 \mathrm{~S}$. Enteritidis strains that showed resistance, 43 (67.2\%) were resistant to two or more antimicrobial agents. Twenty-one (32.8\%) strains were resistant to only one of the antimicrobial agents, 14 to tetracycline, three to nalidixic acid, three to nitrofurantoin, and one to gentamycin. These antimicrobial resistance levels suggest a high occurrence of tetracycline resistant $\mathrm{S}$. Enteritidis strains and resistance to two or more antimicrobial agents.
\end{abstract}

Keywords: poultry, Salmonella Enteritidis, antimicrobial resistance

\section{REFERÊNCIAS BIBLIOGRÁFICAS}

ANTUNES, P.; RÉU, C.; SOUZA, J.C. et al. Incidence of Salmonella from poultry products and their susceptibility to antimicrobial agents. Int. J. Food Microbiol., v.82, p.97-1003, 2003.

BAUMLER, A.J.; HARGIS, B.M.; TSOLIS, R.M. Trancing the origins of Salmonella outbreaks. Science, v.287, p.50-52, 2000.
BOKANYI Jr., R.P.; STEPHENS, J.F.; FOSTER, D.N. Isolation and characterization of Salmonella from broiler carcasses or parts. Poult. Sci., v.69, p.592-598, 1990 .

BRASIL. Ministério da Agricultura, Pecuária e Abastecimento. Secretaria de Defesa Agropecuária. Portaria $\mathrm{n}^{\circ} 126$, de 06 de novembro de 1995. Normas para diagnóstico das salmoneloses aviárias. Anexo I - Descrição das técnicas/métodos bacteriológicos e sorológicos para diagnóstico das 
salmoneloses aviárias. $(S$. Enteritidis, $S$. Gallinarum, $S$. Pullorum e $S$. Typhimurium). Diário Oficial da República Federativa do Brasil, Brasília, DF, 06 nov.1995, Seção 1, n.212, p.17694-17698.

CARDOSO, M.O.; RIBEIRO, A.R.; SANTOS, L.R. et al. Antibiotic resistance in Salmonella Enteritidis isolated from broiler carcasses. Braz. J. Microbiol., v.37, p.299-302, 2006.

CARRAMIÑANA, J.J.; ROTA, C.; AGUSTÍN, I. et al. High prevalence of multiple resistance to antibiotics in Salmonella serovars isolated from a poultry slaughterhouse in Spain. Vet. Microbiol, v.104, p.133-139, 2004.

CORTEZ, A.L.L.; CARVALHO, A.C.F.B.; IKUNO, A.A. et al. Resistência antimicrobiana de cepas de Salmonella spp. isoladas de abatedouros de aves. Arq. Inst. Biol., v.73, p.157-163, 2006.

GOREN, E. Combinación de la aplicación de medicamentos y microflora intestinal como uma herramienta em el tratamiento de lãs infecciones por Salmonella enteritidis em Aves. In: CURSO DE ACTUALIZACION SOBRE EL CONTROL Y PREVENCION DE LA INFECCION POR Salmonella enteritidis, 1., 1994, México. Anais... México: ANECA, 1994. p.13-26.

HOFER, E.; SILVA FILHO, S.J.; REIS, E.M.F.R. Prevalência de serovares de Salmonella isolados de aves no Brasil. Pesq. Vet. Bras., v.17, p.55-62, 1997.

KANASHIRO, A.M.I.; STOPPA, G.F.Z.; CARDOSO, A.L.S.P. et al. Serovars of Salmonella spp. isolated from broiler chickens and commercial breeders in diverse regions in Brazil from July 1997 to December 2004. Braz. J. Poult. Sci., v.7, p.195198, 2005.

MALORNY, B.; SCHROETER, A.; HELMUTH, $\mathrm{R}$. Incidence of quinolones resistance over the period 1986 to 1998 in veterinary Salmonella isolates from Germany. Antimicrob. Agents Chemother., v.43, p.2278-2282, 1999.

MARIMÓN, J.M.; GOMÁRIZ, M.; ZIGORRAGA, C. et al. Incresing prevalence of quinolone resistance in human nontyphoid Salmonella enterica isolates obtained in spain from 1981 to 2003. Antimicrob. agents chemother., v.48, p.27893793, 2004.

PERFOMANCE standards for antimicrobial disk susceptibility tests. Approved standard. 8.ed. Wayne: NCCLS, 2003 (document M2-A8).
PEZZELLA, C.; RICCI, A.; DIGIANNATALE, E. et al. Tetracycline and streptomycin resistance genes, transposons, and plasmids in Salmonella enterica isolated from animals in italy. Antimicrob. Agents Chemother., v.48, p.903-908, 2004.

PIDDOCK, L.J.V. Fluoroquinolone resistance in Salmonella serovars isolated from human and food animals. FEMS Microbiol. Rev., v.26, p.3-16, 2002.

POMEROY, B.S.; NAGARAJA, K.V. Fowl Typhoid. In: CALNEK, B.W. (Ed). Disease of poultry. 9.ed. Ames: Iowa State University, 1991. p.87-99.

POPPE, C.; McFADDEN, K.S.; DEMCZUK, W.H.B. Drug resistance, plasmids, biotypes and susceptibility to bacteriophages of Salmonella isolated from poultry in Canada. Int. J. Food Microbiol., v.30, p.325-344, 1996.

RIBEIRO, A.R.; KELLERMANN, A.; SANTOS, L.R., et al. Salmonella spp. in raw broiler parts: occurrence, antimicrobial resistence profile and phage typing of the Salmonella Enteritidis isolated. Braz. J. Microbiol., v.38, p.296-299, 2007.

RUIZ, J. Mechanisms of resistance to quinolones: target alterations, decresead accumulation and DNA gyrase protection. J. Antimicrob. Chemother., v.51, p.1109-1117, 2003.

SNOEYENBOS, G.H.; WILLIAMS, J.E. Salmonellosis. In: CALNEK, B.W. (Ed). Diseases of Poultry. 9.ed. Ames: Iowa State University, 1991. p.72-73.

SOLARI, C.A.; REIS, E.M.F.; COSTA, R.G., et al. Caracterização dos sorovares de Salmonella isolados de aves de diferentes estados no qüinqüênio 1992-96. In: CONGRESSO BRASILEIRO DE MICROBIOLOGIA, 19., 1997, Rio de Janeiro. Anais... Rio de Janeiro, 1997. p.126. (Resumo).

THRELFALL, E.J.; WARD, L.R.; SKINNER, J.A., et al. Antimicrobial drug resistance in nontyphoidal salmonellas from humans in England and Wales in 1999: decrease in multiple resistance in Salmonella enterica serotypes Typhimurium, Virchow, and Hadar. Microb. Drug Resist., v.6, p.319-325, 2000 .

TURNIDGE, J. Antibiotic use in animals prejudices, perceptions and realities. J. Antimicrob. Chemother., v.53, p.26-27, 2004. 